\title{
Is Ki-67, keratin 16, involucrin, and filaggrin immunostaining sufficient to diagnose inflammatory linear verrucous epidermal nevus? A report of eight cases and a comparison with psoriasis vulgaris*
}

\author{
Jing Peng ${ }^{1}$ \\ Pei-Pei Yang ${ }^{1}$
}

\author{
Shu-Bin Sun ${ }^{2}$ \\ Yi-Ming Fan $^{1}$
}

DOI: http:/ / dx.doi.org/10.1590/abd1806-4841.20176263

\begin{abstract}
Inflammatory linear verrucous epidermal nevus and linear psoriasis are sometimes hard to differentiate clinically and pathologically. Although immunohistochemical expression of keratin 10 (K10), K16, Ki-67, and involucrin may be useful for differentiating both entities, these results have been reported in only a few cases. We collected data from 8 patients with inflammatory linear verrucous epidermal nevus, 11 with psoriasis vulgaris, and 8 healthy controls and evaluated immunohistochemical expression of Ki-67, K16, involucrin, and filaggrin among them. Ki-67 and K16 overexpression was similar in inflammatory linear verrucous epidermal nevus and psoriasis vulgaris compared with normal skin. Although staining for involucrin showed discontinuous expression in parakeratotic regions in 4 inflammatory linear verrucous epidermal nevus cases, it was continuous in the other 4 cases and in all psoriasis vulgaris cases. Filaggrin expression was present in hyperkeratotic regions but scarce in parakeratotic areas in both inflammatory linear verrucous epidermal nevus and psoriasis vulgaris. The immunostaining pattern of Ki-67, K16, involucrin, and filaggrin may be insufficient to discriminate inflammatory linear verrucous epidermal nevus from psoriasis vulgaris.
\end{abstract}

Keywords: Keratinocytes; Keratin-16; Ki-67 Antigen; Psoriasis

\section{INTRODUCTION}

Inflammatory linear verrucous epidermal nevus (ILVEN) is a rare variant of verrucous epidermal nevus that usually occurs in infancy and rarely in adulthood. It is characterized clinically by pruritic, erythematous, verrucous plaques following Blaschko lines, and pathologically by alternating parakeratosis with hypogranulosis and orthokeratosis with hypergranulosis. ${ }^{1}$ ILVEN may sometimes be hard to distinguish from linear psoriasis because of their clinicopathological overlap and concomitance. ${ }^{2-5}$

Although immunohistochemical expression of keratin 10 (K10), K16, Ki-67 and involucrin may be useful for differentiating ILVEN from linear psoriasis, these results have been reported in only a few ILVEN cases. ${ }^{2,6-9}$ We tried to evaluate the expression of Ki-67, $\mathrm{K} 16$, involucrin, and filaggrin for the differential diagnosis between ILVEN and psoriasis vulgaris (PV).

\section{MATERIALS AND METHODS}

This study collected data from 8 untreated ILVEN patients, $11 \mathrm{PV}$ patients, and 8 healthy controls, but no cases of linear psoria- sis. These patients were diagnosed based on clinicopathological features ${ }^{10}$ and not associated with systemic disorders. ILVEN patients had no personal and family histories of psoriasis. PV lesional area was $<10 \%$ of the body surface area.

We performed immunohistochemistry on paraffin-embedded sections. Primary antibodies included goat anti-K16 (SC-49176, 1:200; Santa Cruz Biotechnology, CA, USA), mouse anti-involucrin (SC-21748, 1:200; Santa Cruz), filaggrin (SC-66192, 1:200; Santa Cruz), and rabbit anti-Ki-67 (RMA-0542, prediluted; Zhongshan Jinqiao Biotechnology Co Ltd, Beijing, China). Epidermal Ki-67+ cells were reckoned in 5 different fields at $\times 200$ magnification and expressed in mm of the basement membrane. ${ }^{11}$ The data were analyzed using ANOVA with Dunnett T3 test.

\section{RESULTS}

Clinical data of 8 ILVEN cases are listed in table 1. Mean age and disease course were $36.6 \pm 25.0$ and $20.1 \pm 13.3$ years, respectively. All patients presented with localized or linear verrucous papules, nod-

\section{Received on 14.07.2016.}

Approved by the Advisory Board and accepted for publication on 24.12.2016.

Work performed at the Department of Dermatology, Affiliated Hospital of Guangdong Medical University - Zhanjiang, Guangdong, China

Financial support: none.

Conflict of interest: none.

\section{Department of Dermatology, Affiliated Hospital of Guangdong Medical University, Zhanjiang, Guangdong, China}

Department of Dermatology, Dongguan 3rd People's Hospital - Dongguan, Guangdong, China

(C2017 by Anais Brasileiros de Dermatologia 


\begin{tabular}{lllll} 
Case & Gender & Age/duration & Lesional distribution & Involucrin expression \\
\hline $\mathbf{1}$ & Male & $55 / 30$ years & Right cheek, neck, axilla, and inner thigh & Discontinuous \\
2 & Male & $5 / 0.2$ years & Perianal area & Discontinuous \\
3 & Male & $14 / 13.7$ years & Left lower lip & Discontinuous \\
4 & Female & $8 / 5$ years & Left forearm, thigh, and leg & Discontinuous \\
5 & Male & $41 / 20$ years & Left inner thigh and upper leg & Continuous \\
$\mathbf{6}$ & Male & $42 / 25$ years & Right thigh, leg, and dorsal foot & Continuous \\
7 & Female & $73 / 40$ years & Left temple & Continuous \\
8 & Male & $55 / 27$ years & Left inner and anterior thigh and posterior leg & Continuous
\end{tabular}
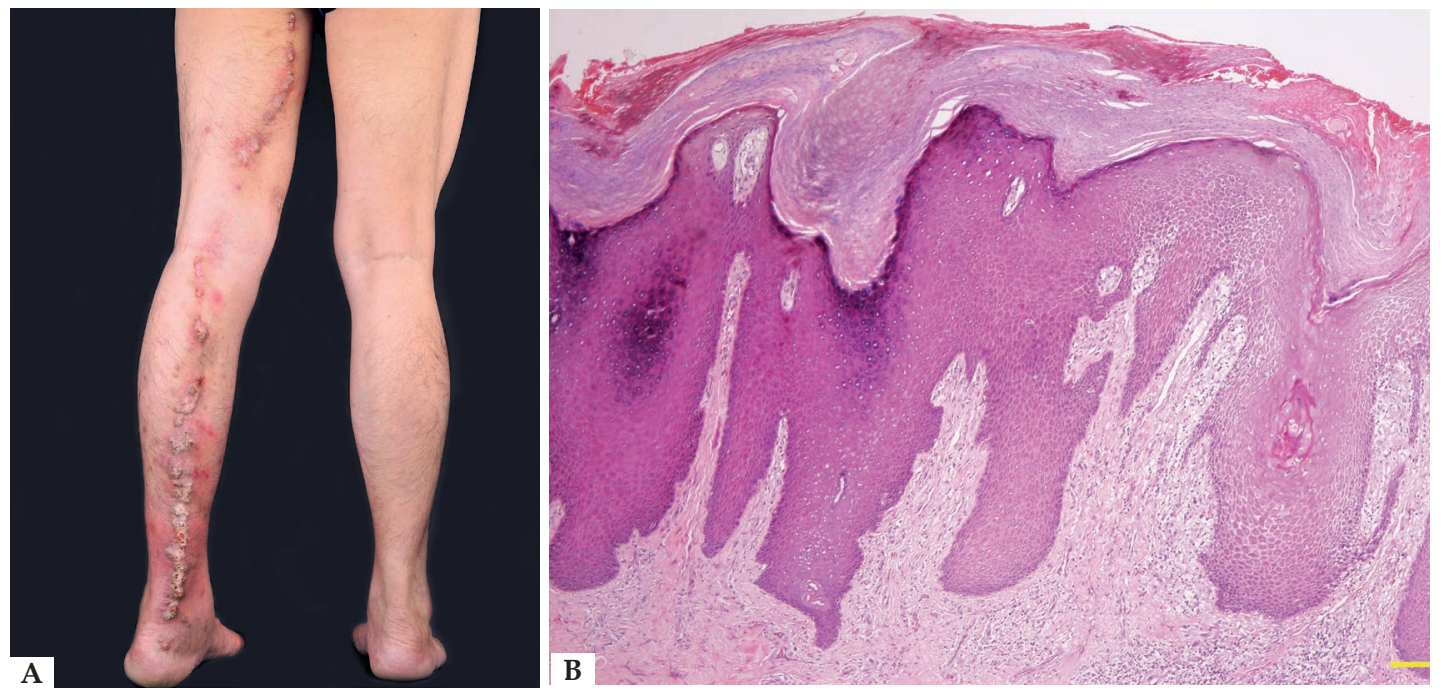

FIGURE 1:

Clinicopathological presentation of ILVEN in case 8.

A. Linearly arranged brown verrucous nodules and plaques along the left inner thigh and posterior leg.

B. Epidermal acanthosis, alternating parakeratosis with agranulosis, orthokeratosis with hypergranulosis, and perivascular lymphocytic infiltration (Hematoxylin \& eosin scale bar $=200 \mu \mathrm{m}$ )

ules, or plaques (Figure 1A). All patients but one had intense pruritus. Typical pathological features were epidermal acanthosis, alternating parakeratosis with hypogranulosis, orthokeratosis with hypergranulosis, and perivascular lymphocytic dermal infiltration (Figure 1B).

Eleven PV cases comprised 8 males and 3 females. The average age and disease course were $34.6 \pm 15.8$ and $3.4 \pm 4.3$ years, respectively. Eight controls included 7 males and 1 female, with average age of $39.9 \pm 9.8$ years.

Ki-67 was expressed in the basal and parabasal keratinocyte layers of the epidermis, pilosebaceous units, and in some eccrine duct cells. Epidermal Ki-67 ${ }^{+}$cells in ILVEN $(6.86 \pm 2.34)$ and PV $(5.31 \pm 2.54)$ were higher than those of normal skin $(1.78 \pm 0.83)(p<0.01)$, but the difference between ILVEN and PV was not significant $(p>0.05)$.

K16 expression was absent in the epidermis of the normal skin. However, we observed K16 expression from the suprabasal layer to the horny layers of parakeratotic regions, while its expression was absent or present only in the spinous layers of hyperkeratotic regions in ILVEN and PV patients.

Filaggrin was expressed in the horny and granular layers in normal skin. However, its expression was evident in the lower horny and granular layers of hyperkeratotic areas but deficient in parakeratotic areas in ILVEN and PV patients.
We detected involucrin expression in the granular and upper spinous layers in normal skin, but it extended into the middle spinous layer in ILVEN and PV patients. Involucrin showed discontinuous staining (e.g., increased expression in hyperkeratotic regions but absent in parakeratotic regions) in 4 ILVEN cases, but continuous staining in the other ILVEN cases and in all PV cases (Table 1, Figures 2 and 3).

\section{DISCUSSION}

Expression patterns for Ki-67 and K16 are upregulated in PV cases, and their expression is documented in a few ILVEN cases. Incremental $\mathrm{Ki}-67^{+}$cells and local $\mathrm{K} 16$ staining were seen in 4 ILVEN cases. ${ }^{6,11,12} \mathrm{Ki}-67$ expression tended to be lower in 3 ILVEN cases than in 4 psoriatic cases but with a remarkable overlap. ${ }^{7}$ In a study, a female patient initially considered to have linear psoriasis was finally diagnosed as ILVEN based on a lower Ki-67 expression. ${ }^{2}$ However, our study uncovered an analogous overexpression of Ki-67 and K16 in ILVEN and PV patients. Diffuse and intense K16 staining was observed from the suprabasal layer to the horny layers of parakeratotic regions, while its expression was absent or weak in the spinous layers of hyperkeratotic regions in both ILVEN and PV cases. Hence, our results reveal that epidermal hyperproliferation is 

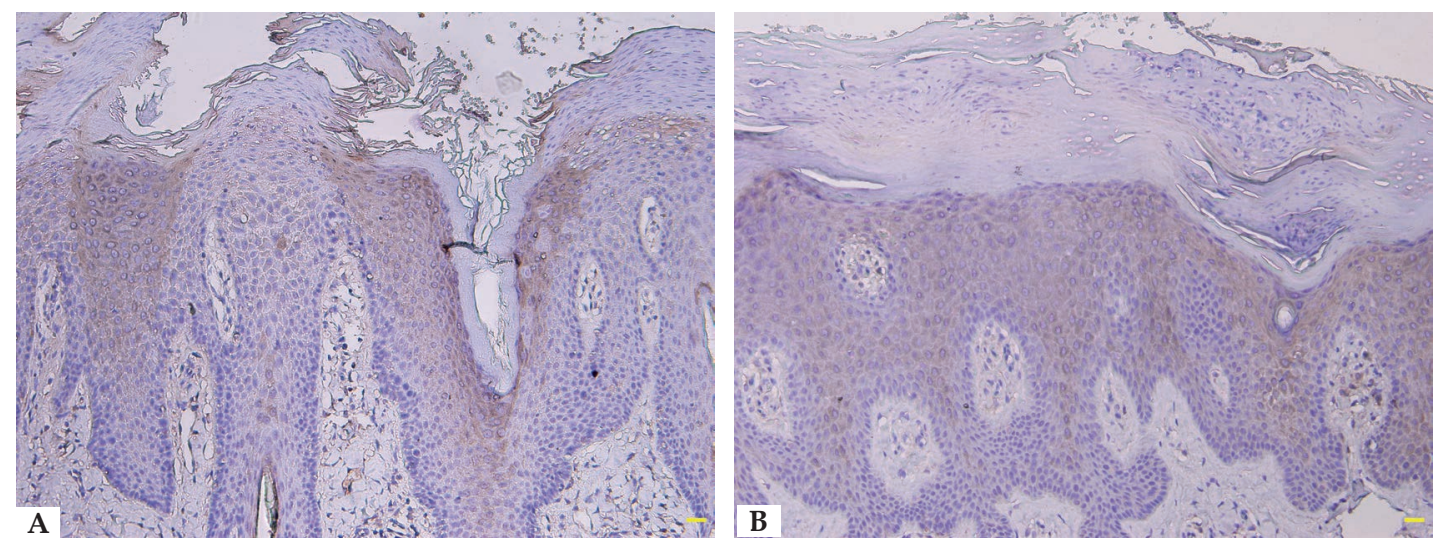

Figure 2:

A. Discontinuous involucrin immunostaining in ILVEN (immunostaining, scale bar $=100 \mu \mathrm{m}$ ). B. Continuous involucrin immunostaining in ILVEN (immunostaining, scale bar $=$ $100 \mu \mathrm{m})$

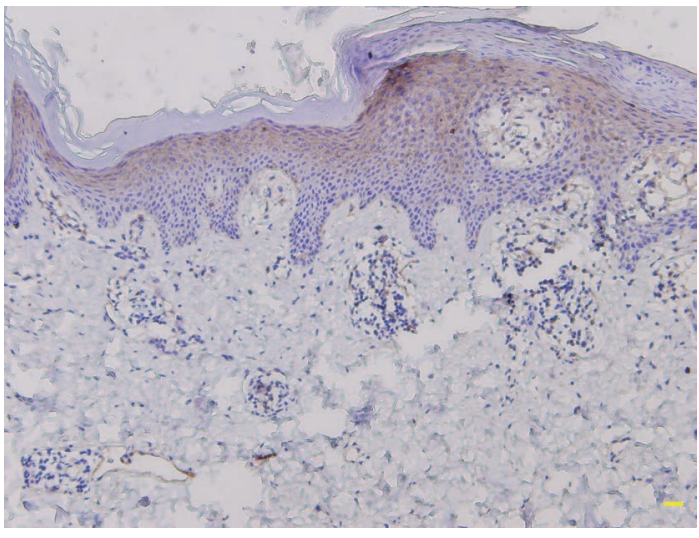

Figure 3: Continuous involucrin immunostaining in psoriasis vulgaris (immunostaining, scale bar $=100 \mu \mathrm{m}$ )

comparable in both disorders and Ki-67 is not a potential marker to distinguish them. Since K16-related inflammatory response could be involved in epidermal hyperproliferation, ${ }^{13}$ disparate K16 distribution and intensity between parakeratotic and hyperkeratotic areas might suggest distinct alterations of keratinocytic activation and proliferation.
Involucrin overexpression and filaggrin down-expression are existent in psoriatic lesions. ${ }^{14-16}$ Involucrin expression was evident in orthokeratotic regions, but scanty in parakeratotic regions in 4 ILVEN cases, while suprabasal keratinocytes expressed involucrin in parakeratotic areas of psoriasis. ${ }^{8,9}$ Some authors considered that involucrin immunostaining might be valuable to distinguish between ILVEN and psoriasis. ${ }^{8,9,17}$ However, we observed that involucrin staining was discontinuous in parakeratotic regions in 4 ILVEN cases, while its staining was continuous in the other ILVEN cases and in all PV cases, suggesting that involucrin immunostaining may be unreliable for differentiating both entities. Furthermore, filaggrin expression was present in hyperkeratotic regions but scarce in parakeratotic areas in ILVEN and PV. These results demonstrate that both ILVEN and PV might have an immature epidermal cornification. ${ }^{14}$ In parakeratotic areas, lack of filaggrin expression can result from an absent granular layer because its expression is restricted to horny and granular layers in normal skin; the absence of involucrin staining, however, might be due to insufficient production of cytokines since some cytokines can reinforce involucrin expression in both normal and psoriatic keratinocytes. ${ }^{14}$

In summary, our results demonstrate that immunostaining patterns for Ki-67, K16, involucrin, and filaggrin may be insufficient to differentiate between ILVEN and PV. $\square$ 


\section{REFERENCES}

1. Khachemoune A, Janjua SA, Guldbakke KK. Inflammatory linear verrucous epidermal nevus: a case report and short review of the literature. Cutis. 2006;78:261-7.

2. Chu GY, Hu SC, Lan CC. Unusual presentation of inflammatory linear verrucous epidermal naevus mimicking linear psoriasis successfully treated with oral retinoid. J Eur Acad Dermatol Venereol. 2015;29:2255-7.

3. Agarwal US, Besarwal RK, Gupta R, Agarwal P. Inflammatory linear verrucous epidermal nevus with psoriasiform histology. Indian J Dermatol. 2014;59:211.

4. Ozdemir M, Mevlitoğlu I, Balevi A. Acitretin narrow-band TL-01 phototherapy but not etanercept treatment improves a localized inflammatory linear verrucous epidermal naevus with concomitant psoriasis. J Eur Acad Dermatol Venereol. 2009;23:1453-4.

5. Özdemir M, Balevi A, Esen H. An inflammatory verrucous epidermal nevus concomitant with psoriasis: treatment with adalimumab. Dermatol Online J. 2012;18:11

6. de Jong E, Rulo HF, van de Kerkhof PC. Inflammatory linear verrucous epidermal naevus (ILVEN) versus linear psoriasis. A clinical, histological and immunohistochemical study. Acta Derm Venereol. 1991;71:343-6.

7. Vissers WH, Muys L, Erp PE, de Jong EM, van de Kerkhof PC. Immunohistochemical differentiation between inflammatory linear verrucous epidermal nevus (ILVEN) and psoriasis. Eur J Dermatol. 2004;14:216-20.

8. Ito M, Shimizu N, Fujiwara H, Maruyama T, Tezuka M. Histopathogenesis of inflammatory linear verrucose epidermal naevus: histochemistry, immunohistochemistry and ultrastructure. Arch Dermatol Res. 1991;283:491-9.

9. Ferreira FR, Di Chiacchio NG, Alvarenga ML, Mandelbaum SH. Involucrin in the differential diagnosis between linear psoriasis and inflammatory linear verrucous epidermal nevus: a report of one case. An Bras Dermatol. 2013;88:604-7.

10. Mobini N, Toussaint S, Kamino H. Noninfectious erythematous, papular, and squamous diseases. In: Elder DE, Elenitas R, Johnson Jr BL, Murphy GF, Xu G, editors. Lever's Histopathology of the Skin. 10th ed. Philadelphia: Lippincott Williams \& Wilkins; 2008. p. 174-181, 194-195.

11. Bovenschen HJ, Seyger MM, Van de Kerkhof PC. Plaque psoriasis vs. atopic dermatitis and lichen planus: a comparison for lesional T-cell subsets, epidermal proliferation and differentiation. Br J Dermatol. 2005;153:72-8.

12. Bhawan J, Bansal C, Whren K, Schwertschlag U; IL-11 Psoriasis Study Group. K16 expression in uninvolved psoriatic skin: a possible marker of pre-clinical psoriasis. J Cutan Pathol. 2004;31:471-6.

13. Lessard JC, Piña-Paz S, Rotty JD, Hickerson RP, Kaspar RL, Balmain A, et al. Keratin 16 regulates innate immunity in response to epidermal barrier breach. Proc Natl Acad Sci U S A. 2013;110:19537-42.

14. Chen JQ, Man XY, Li W, Zhou J, Landeck L, Cai SQ, et al. Regulation of involucrin in psoriatic epidermal keratinocytes: the roles of ERK $1 / 2$ and GSK-3 $\beta$. Cell Biochem Biophys. 2013;66:523-8.

15. lizuka H, Takahashi H, Honma M, Ishida-Yamamoto A. Unique keratinization process in psoriasis: late differentiation markers are abolished because of the premature cell death. J Dermatol. 2004;31:271-6

16. Tschachler E. Psoriasis: the epidermal component. Clin Dermatol. 2007:25:589-95.

17. Ginarte $\mathrm{M}$, Fernández-Redondo $\mathrm{V}$, Toribio J. Unilateral psoriasis: a case individualized by means of involucrin. Cutis. 2000;65:167-70

\author{
MAILING ADDRESS: \\ Yi-Ming Fan \\ Department of Dermatology, \\ Affiliated Hospital of Guangdong Medical University \\ Zhanjiang, \\ Guangdong, 524001, China \\ E-mail:ymfan1963@163.com
}

How to cite this article: Peng J, Sun SB, Yang PP, Fan YM. Is Ki-67, keratin 16, involucrin, and filaggrin immunostaining sufficient to diagnose inflammatory linear verrucous epidermal nevus? A report of eight cases and a comparison with psoriasis vulgaris. An Bras Dermatol. 2017;92(5):682-5. 